\title{
ANÁLISE QUALITATIVA DA DIREÇÃO DA CORRENTE LONGITUDINAL ENTRE 2004 E 2008 NA PRAIA DE TRAMANDAÍ, RS, BRASIL
}

\author{
QUANTITATIVE ANALYSIS OF THE LONGSHORE CURRENT'S \\ DIRECTION BETWEEN 2004 AND 2008 AT TRAMANDAÍ BEACH, \\ RS, BRAZIL
}

Gabriela B. Jung

Universidade Federal do Rio Grande do Sul, Av. Bento Gonçalves, 9500, Campus do Vale - e-mail: jung oceano@yahoo.com.br

Elírio Ernestino Toldo Jr. Universidade Federal do Rio Grande do Sul, Av. Bento Gonçalves, 9500, Campusdo Vale - e-mail: toldo@ufrgs.br

\begin{abstract}
Resumo
Análises qualitativas da direção superficial da corrente longitudinal, na praia de Tramandaí, RS, de 2004 a 2008, mostram a ocorrência anual de correntes sem uma única direção preferencial, sendo hora para nordeste, hora para sudoeste. A análise mensal dos registros mostra que fevereiro e março apresentaram maior bidirecionalidade que outros meses, com frequências de ocorrência semelhante de correntes para nordeste e para sudoeste. Embora o fluxo apresente ambas as direções em todos os meses, predominantemente no verão e primavera o fluxo é para sudoeste devido à ocorrência de intensos ventos alísios de nordeste, enquanto no outono e no inverno, devido às frentes frias, correntes para nordeste são mais frequentes. Comparações entre as direções simultâneas do vento, das ondas e das correntes, mostram que o ângulo de incidência das ondas é o principal fator influenciador da direção superficial da corrente.
\end{abstract}

Palavras-chave: hidrodinâmica, zona de surfe, correntes litorâneas.

\begin{abstract}
Qualitative analysis of the surface longshore current direction at Tramandaí Beach, RS, from 2004 to 2008, show an annual occurrence without one preferential direction, being sometimes to NE, sometimes to SW. Monthly analysis from data showed february and march with larger bidirectionality than other months, with similar NE and SW occurrence frequencies. Although the flow shows both directions in all months, especially during summer and spring the flow is SW, due to intense NE winds, while during fall and winter, due to cold front, NE currents are more frequent. Comparisons between simultaneous wind, wave and current data, indicate that the wave's incidence angle is the most important variable to determine longshore current's direction.
\end{abstract}

Keywords: hydrodynamics, surf zone, longshore currents. 


\section{Introdução}

Correntes longitudinais são formadas quando as ondas apresentam incidência oblíqua à linha de praia, provocando um fluxo em direção à costa de tensão de radiação longitudinal. A intensidade destas correntes é dependente da altura das ondas e do ângulo de incidência das mesmas, apresentando relação diretamente proporcional (Longuet-Higgins, 1970).

Existe uma relação direta entre direção das ondas na arrebentação e direção do vento, sendo constatado que o vento pode ser um dos principais fatores a ditar a direção das ondas na arrebentação e por consequência, a corrente na costa (Lanfredi \& Framiñan, 1986).

Hubertz (1986), analisou a direção da corrente superficial, comparando-a com a direção dos ventos e ondas incidentes, e notou concordância entre três variáveis na maior parte do tempo. Foi encontrada discordância entre os dados apenas em situações de propagação de ondas na direção contrária da direção do vento.

Fontoura (2004), encontrou resultados semelhantes, onde as direções da corrente mostram-se quase integralmente dependentes da direção e intensidade da componente longitudinal do vento na costa. Alterações na direção do vento provocam pronta resposta da direção da corrente, que busca imediatamente acompanhar a direção do vento. Exceções ocorrem quando a componente do vento é muito pequena ou nula, neste caso a corrente passa a ser governada pela direção das ondas na arrebentação.

Nas praias do Rio Grande do Sul a corrente mostra duas direções predominantes de propagação, para SW e para NE. Fluxos para nordeste predominam durante o inverno, enquanto no verão prevalecem correntes para sudoeste (Alvarez et al., 1981).

Tozzi (1999), a partir de dados obtidos em cinco anos de observações visuais diárias do estado do mar ao largo da Praia do Cassino, mostrou que as correntes para SW são predominantes em todas as épocas do ano, muito embora as correntes mais intensas tenham sido observadas seguindo para nordeste. Calliari et al. (2005), através de dados coletados por Puvs, observou que ventos intensos provocam aumento na altura da ondas e na velocidade da corrente longitudinal.

Na praia de Tramandaí, litoral norte do Rio Grande do Sul, Nicolodi et al. (2000), com base numa série de dados observados em 1996 e 1997, encontrou o mesmo padrão bidirecional da direção da corrente longitudinal superficial observado por Alvarez et al. (1981).

\section{Área de estudo}

A praia de Tramandaí (Figura 1), localizada no litoral norte do Rio Grande do Sul, dispõe de facilidades para o fundeio de equipamentos oceanográficos a partir de uma plataforma de pesca, que possibilitou a aquisição de dados instantâneos de velocidades e direção da corrente longitudinal. A plataforma apresenta comprimento de $365 \mathrm{~m}, 8 \mathrm{~m}$ de largura e estrutura em forma de T (Figura 2). Sob condições de ondulação significativa esta estrutura atravessa toda a zona de surfe.

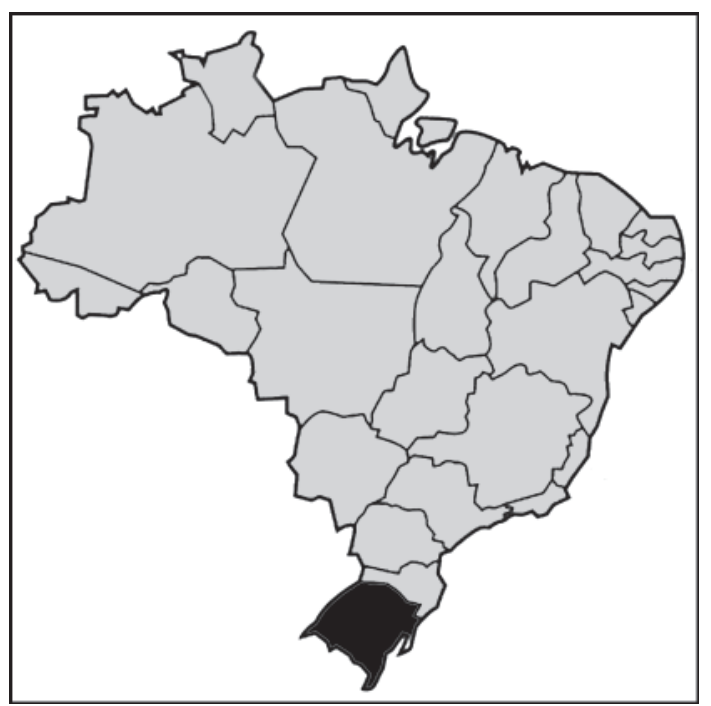

Figura 1 - Localização da área de estudo no litoral norte do estado do Rio Grande do Sul (Google Earth ${ }^{\circledR}$ ).

O litoral norte está inserido na extensa costa do estado com orientação uniforme NE-SW e leve sinuosidade ao longo dos seus $615 \mathrm{~km}$ (Figura 1). Toda esta área é constituída por depósitos quaternários inconsolidados que não recebem contribuições de areias modernas, pois toda carga de tração transportada pela rede de drenagem interior é retida nas lagunas e outros ambientes costeiros, como por exemplo, a Lagoa dos Patos e a Lagoa Mirim. A Plataforma Continental é parte de uma ampla e passiva margem, com mais de 150 $\mathrm{km}$ de extensão, profundidades máximas variando entre 100 e $140 \mathrm{~m}$ e suave declividade da ordem de $0,06^{\circ}$. A antepraia é ampla e rasa com limite externo na profundidade de $10 \mathrm{~m}$, onde predominam depósitos arenosos (Toldo et al., 2006). A Praia de Tramandaí possui um alinhamento de aproximadamente $110^{\circ}$.

Incidem sobre a costa ondulações geradas no Oceano Atlântico Sul e vagas geradas pelos fortes ventos locais de verão e primavera, provenientes de NE. Exceto pela passagem das frentes frias de S e SE, a agitação marítima é caracterizada por ondas de média a elevada energia, com altura significativa de $1,5 \mathrm{~m}$ e período entre 7 e $9 \mathrm{~s}$. A maré astronômica é semi-diurna, com amplitude média de $0,25 \mathrm{~m}$, sendo que a maré meteorológica pode alcançar 1,20m (Almeida et al., 1997). A profundidade de fecha- 
mento é estimada em 7,5m, calculada com base em dois conjuntos de dados de ondas coletados nos anos de 1963 e 1996 no Litoral Norte do estado (Almeida et al., 1999).
Consequentemente, o transporte e a deposição dos sedimentos ao longo da costa são primariamente dominados pela ação da onda.

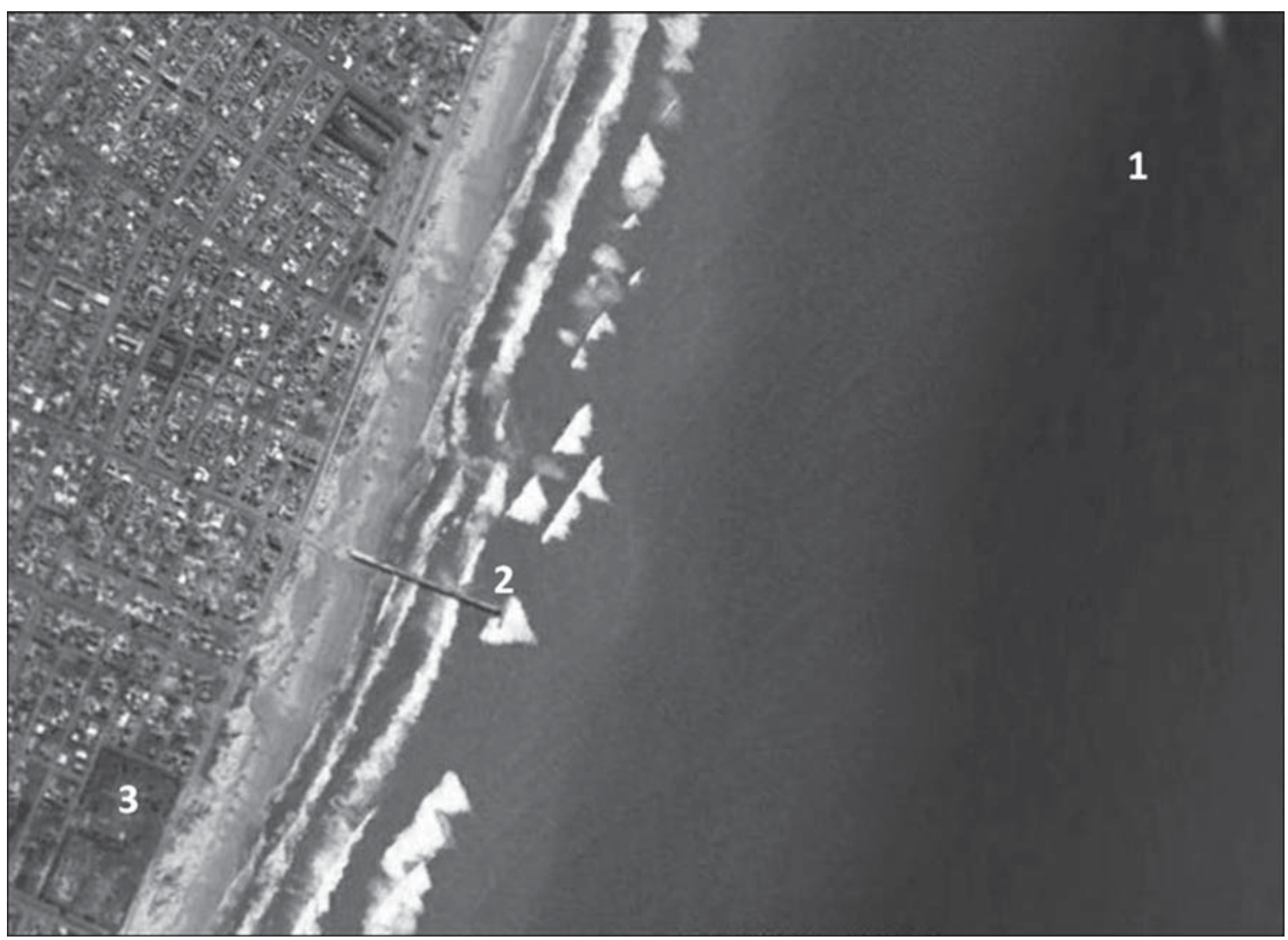

Figura 2 - Localização da praia de Tramandaí no litoral norte do estado. Na imagem Google Earth ${ }^{\circledR}$ estão assinalados os pontos de aquisição dos dados: corrente longitudinal na plataforma de pesca (2), vento na estação meteorológica (3), e local de fundeio do ondógrafo (1).

\section{Metodologia}

Medidas diárias da direção da corrente superficial são realizadas na plataforma de pesca de Tramandaí, às 08:00, 12:00 e 16:00 horas. A direção da corrente é observada no canal principal, entre o primeiro e o segundo banco arenoso (Figura 2), através do espalhamento da espuma associada a quebra da onda.

Ao contrário da convenção adotada na Oceanografia, cuja direção da corrente aponta o sentido no qual esta se desloca (para nordeste ou para sudoeste), as observações realizadas na plataforma são no sentido contrário, de onde a corrente vem (de nordeste ou de sudoeste), convenção utilizada entre os pescadores. Portanto, neste trabalho, a direção dos dados cedidos foi invertida, a fim de enquadrar-se na metodologia científica.

Foram analizados dados mensais de 2004 a 2008, sendo que para 2007 os dados disponíveis são de janeiro a junho, e para 2008 de janeiro a maio.

De novembro de 2006 a junho de 2007, o ondógrafo modelo WaveRider da Datawell ${ }^{\circledR}$ foi fundeado no ponto $29^{\circ} 59^{\prime} 58^{\prime} \mathrm{S}, 50^{\circ} 06^{\prime} 09^{\prime} \mathrm{W}$, a $17 \mathrm{~m}$ de profundidade. O equipamento coletou dados de altura, período e ângulo de incidência das ondas.

Deste modo, com os dados concomitantes de onda coletados pelo ondógrafo, de direção da corrente superficial, registrados na plataforma, e de vento coletados pela Estação Meteorológica de Tramandaí, é possível verificar quais são as variáveis controladoras da direção da corrente.

\section{Resultados e Discussões}

A (Figura 3) apresenta os dados de vento durante o período de fundeio do ondógrafo. 


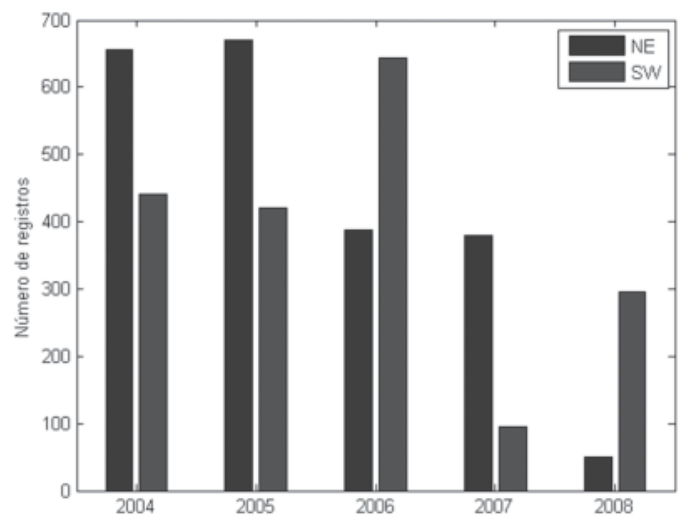

Figura 3 - Distribuição da direção do vento durante o período de fundeio do ondógrafo, entre novembro de 2006 e junho de 2007.

Os dados mostram maior predomínio dos ventos de NE de novembro a fevereiro, bidirecionalidade em março e abril, e maior ocorrência dos ventos de SW em maio e junho. $\mathrm{O}$ padrão apresentado é o esperado para a região, com ventos de NE durante o verão e outono, SW durante o inverno.

A figura 4 apresenta o número de registros de correntes para NE e para SW de 2004 a 2008.

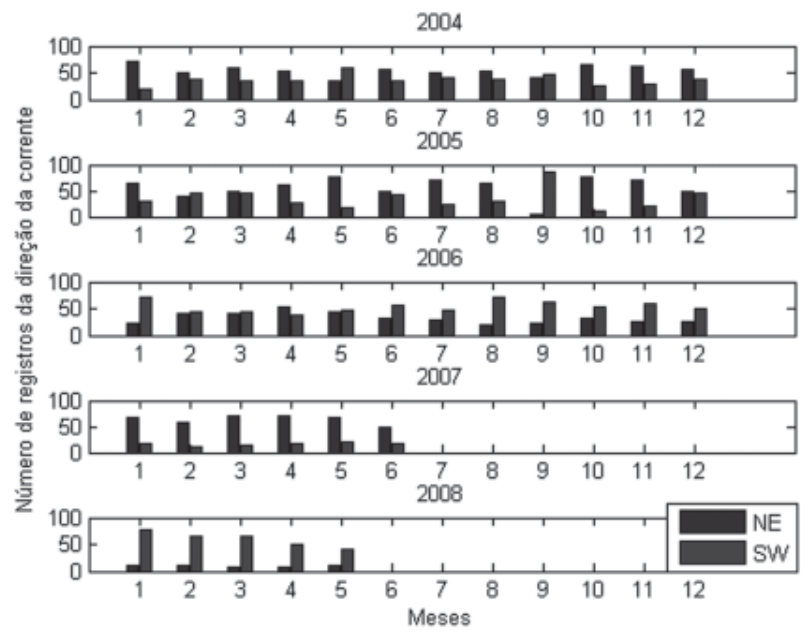

Figura 4 - Histograma de registros anuais da direção da corrente superficial entre os anos de 2004 e 2008.

Observa-se a propagação das correntes para ambas as direções em todos os anos, havendo predomínio para NE em 2004 e 2005, e para SW em 2006. A variação anual da direção da corrente também foi observada por Nicolodi et al. (2000), cujos dados apontam a prevalência de correntes para SW em 1996 e 1997, e para NE em 1998 e 1999.

Em 2007 houve predomínio de correntes para NE, visto que o período de aquisição de dados foi de janeiro a junho. Correntes para NE são predominantes no outono e no inverno, e para SW no verão e na primavera (Alvarez et al.,
1981). Como os registros de corrente em 2007 são relativos ao período do verão, outono e parte do inverno, é esperada a maior frequência de correntes para SW.

Em 2008 os dados utilizados correspondem aos meses de janeiro a maio. A principal direção observada é SW. Isso ocorre pois as frentes frias são mais frequentes a partir de abril, sendo os ventos de NE predominantes durante o verão, gerando ondas do mesmo quadrante, que formam correntes para SW.

Na figura 5 as direções de correntes, para NE e para $\mathrm{SW}$, foram avaliadas mensalmente.

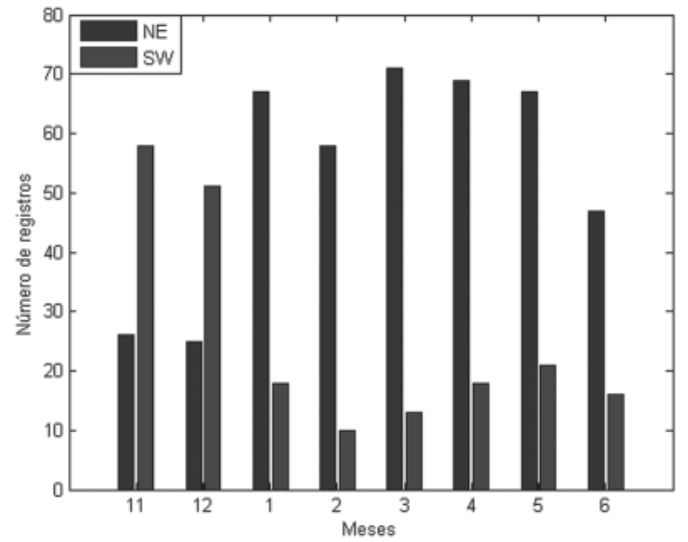

Figura 5 - Registros mensais da direção da corrente entre 2004 e 2008 .

Durante o mês de janeiro de todos os anos analisados houve uma direção de propagação predominante em relação à outra, com maior quantidade de registros para SW em 2006 e 2008, e para NE nos demais anos.

Em fevereiro dos anos 2004, 2005 e 2006 o fluxo mostrou-se bidirecional, para NE e para SW, em frequência de ocorrência muito semelhante. Em 2007 o fluxo foi preferencialmente para NE, enquanto em 2008 foi para SW (Figura 5).

Padrão semelhante foi observado no mês de março, apresentando a mesma bidirecionalidade em 2005 e 2006, e maior ocorrência de correntes para NE em 2004 e 2007, enquanto em 2008 houve predomínio de correntes para SW. De janeiro a março prevalescem os ventos de NE na costa do Rio Grande do Sul, gerando vagas que formam correntes para SW. Os registros para NE podem estar associados às passagens de frentes frias, gerando ondulações e correntes para NE.

Trabalhos anteriores de Alvarez et al. (1981), Fontoura (2004) e Linsiowski (2006), indicam um padrão de ocorrência de correntes para SW nos meses de verão e primavera, e para NE durante os meses de outono e inverno.

No mês de abril entre 2004 e 2007 houve predomínio de correntes para NE, para SW apenas em 2008. 
Maio de 2006 apresentou padrão bidirecional, com fluxos para SW em 2004 e 2008, e para NE em 2005 e 2007. Em junho, o padrão bidirecional ocorreu em 2005 , as correntes para NE predominaram em 2004 e 2007, enquanto em 2006 foi observada maior incidência de correntes para SW.

Em junho e julho de 2006 houve maior ocorrência de correntes para SW. Correntes para NE foram predominantes em junho de 2004 e 2007, e julho de 2005. Em junho de 2005 o padrão foi bidirecional, assim como em julho de 2004.

Em agosto de 2004 e 2005 as correntes para NE foram predominantes, enquanto em 2006 foram observadas mais correntes para SW. Em setembro de 2005 e 2006 o fluxo foi principalmente para SW, e bidirecional em 2004. Em outubro e novembro o comportamento foi semelhante, com maior ocorrência para SW em 2006, e para NE em 2004 e 2005. Enquanto em dezembro de 2004 houve predominância de correntes para NE, para SW em 2006, e bidirecionalidade em 2005 (Figura 5).

As principais forçantes da corrente longitudinal são a altura e o ângulo de incidência das ondas (LonguetHiggins, 1970), portanto, a direção da corrente superficial tende a acompanhar a direção de propagação das ondas na zona de surfe. O vento também influencia a direção da propagação das correntes longitudinais, transferindo momentum para a coluna d'água (Feddersen et al., 2007). Sendo assim, a figura 6 mostra a distribuição da direção da corrente durante os meses de fundeio do ondógrafo, onde em novembro e dezembro as correntes predominantes foram para SW e nos demais meses de fundeio, para NE. A figura 7 representa os parâmetros de onda registrados pelo equipamento.
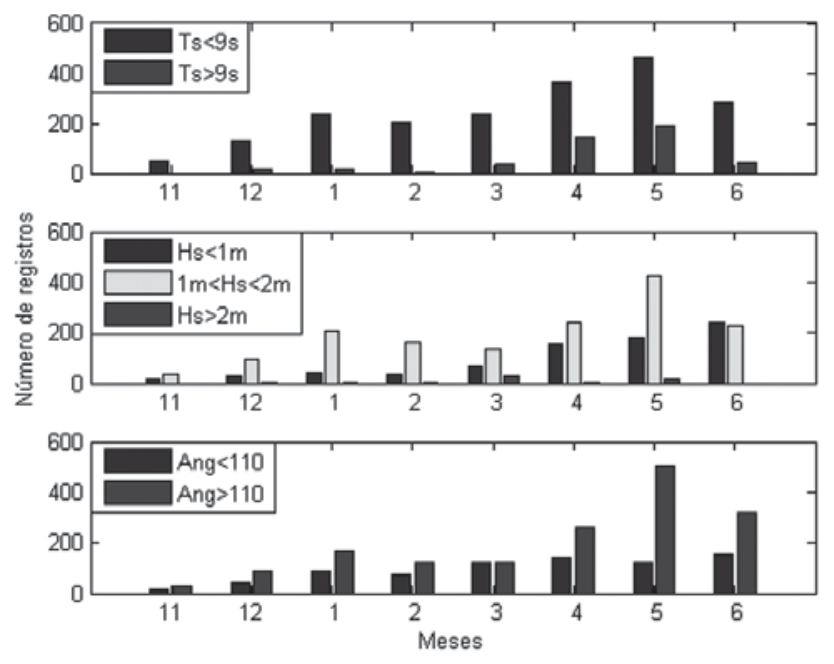

Figura 6 - Distribuição da direção da corrente nos meses de fundeio do ondógrafo, entre dezembro de 2006 e junho de 2007.
Através da figura 7 pode-se inferir que houve o predomínio de vagas em todos os meses, com a presença de ondulações entre março e junho, época em que a passagem das frentes frias é mais frequente e intensa, gerando ondas de maior período. As maiores alturas significativas também foram registradas durante abril maio e junho, relacionadas à maior incidência de ondas do sul.

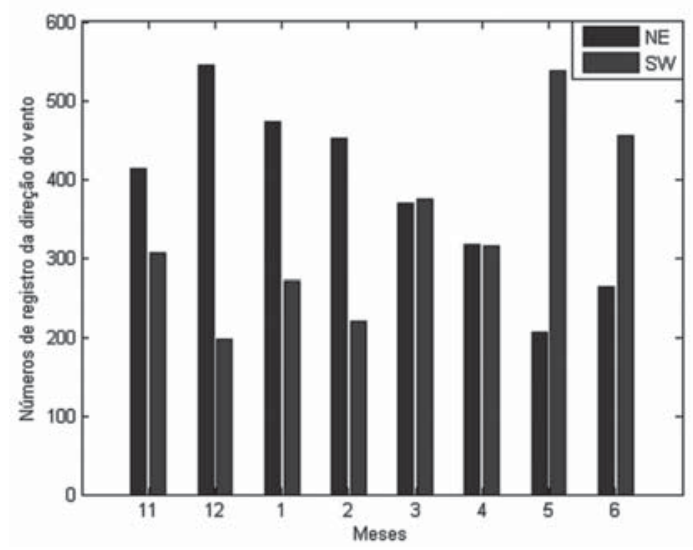

Figura 7 - Distribuição dos parâmetros de onda adquiridos entre novembro de 2006 e junho de 2007, onde $H_{s}=$ altura significativa, $T_{s}=$ período significativo e Ang = ângulo de incidência .

A Tabela 1 apresenta o intervalo de variação dos registros do ondógrafo para cada mês de coleta de dados. Os dados mostram ocorrência de ondulações em todos os meses, com períodos entre 4,1s a 13,1s. Março foi o mês que apresentou ondas com maiores alturas, entre $0,7 \mathrm{~m}$ a $2,93 \mathrm{~m}$, bem como valores mais elevados de ângulo de incidência, variando de $58,92^{\circ}$ a $201,62^{\circ}$.

Tabela 1 - Intervalo de variação dos registros de onda, onde $\mathbf{H}_{\mathrm{s}}=$ altura significativa, $\mathbf{T}=$ período e Ângulo = ângulo de incidência.

\begin{tabular}{|c|c|c|c|}
\hline & Hs (m) & $\mathrm{T}(\mathrm{s})$ & Ângulo (graus) \\
\hline Novembro & $0,52 \mathrm{~m}$ a $1,97 \mathrm{~m}$ & $4,4 \mathrm{~s}$ a $7,2 \mathrm{~s}$ & $79,30^{\circ}$ a 161,63 \\
\hline Dezembro & $0,64 \mathrm{~m}$ a $2,05 \mathrm{~m}$ & $4,9 \mathrm{~s}$ a $10,8 \mathrm{~s}$ & $77,61^{\circ}$ a 187,28 \\
\hline Janeiro & $0,46 m$ a $2,89 m$ & 5,1 s a $10,8 \mathrm{~s}$ & 27,56 a 187,73 \\
\hline Fevereiro & $0,55 \mathrm{~m}$ a $2,02 \mathrm{~m}$ & $4,1 \mathrm{~s}$ a $9,2 \mathrm{~s}$ & $21^{\circ}$ a 182,94 \\
\hline Março & $0,7 \mathrm{~m}$ a $2,93 \mathrm{~m}$ & $4,6 \mathrm{~s}$ a $9,1 \mathrm{~s}$ & 58,92 a 201,62 \\
\hline Abril & $0,64 \mathrm{~m}$ a $2,68 \mathrm{~m}$ & 4,9 s a $13,1 \mathrm{~s}$ & 39,04 a 186,93 \\
\hline Maio & $0,51 \mathrm{~m}$ a $2,32 \mathrm{~m}$ & $5,1 \mathrm{~s} \mathrm{a} 12,4 \mathrm{~s}$ & 38,63 a $201,55^{\circ}$ \\
\hline Junho & $0,19 \mathrm{~m}$ a $1,79 \mathrm{~m}$ & $4,6 \mathrm{~s}$ a $14 \mathrm{~s}$ & 29,98 a 198,02 \\
\hline
\end{tabular}

Para uma avaliação precisa do efeito das ondas e do vento na corrente longitudinal, foram calculadas as frequências de concordância entre estas direções, e da direção do vento e da corrente longitudinal, apenas nos horários dos registros realizados na plataforma de pesca (Tabela 2 ). 
Tabela 2 - Frequência de concordância entre as direções da onda e da corrente, e do vento e da corrente, onde $\mathrm{NE}=$ correntes para $\mathrm{NE}, \mathrm{SW}=$ correntes para $\mathrm{SW}$.

\begin{tabular}{|c|c|c|c|c|}
\hline & \multicolumn{2}{|c|}{ Corrente x Onda } & \multicolumn{2}{c|}{ Corrente x Vento } \\
\cline { 2 - 5 } & NE & SW & NE & SW \\
\hline $\begin{array}{c}\text { Novembro } \\
2006\end{array}$ & 92,3 & 7,69 & 77,77 & 18,66 \\
\hline $\begin{array}{c}\text { Dezembro } \\
2006\end{array}$ & 27,27 & 36,36 & 45,31 & 5,47 \\
\hline $\begin{array}{c}\text { Janeiro } \\
2007\end{array}$ & 34,54 & 18,18 & 60,25 & 11,53 \\
\hline $\begin{array}{c}\text { Fevereiro } \\
2007\end{array}$ & 49,99 & 17,76 & 61,46 & 8,95 \\
\hline $\begin{array}{c}\text { Março } \\
2007\end{array}$ & 47,16 & 9,43 & 52,43 & 10,96 \\
\hline $\begin{array}{c}\text { Abril } \\
2007\end{array}$ & 34,99 & 21,66 & 52,85 & 14,56 \\
\hline $\begin{array}{c}\text { Maio } \\
2007\end{array}$ & 28,91 & 25,08 & 22,52 & 21,17 \\
\hline $\begin{array}{c}\text { Junho } \\
2007\end{array}$ & 31 & 19 & 26,85 & 17,46 \\
\hline
\end{tabular}

\section{Conclusões}

De um modo geral, o padrão proposto nos trabalhos anteriores foi encontrado nesta pesquisa. As direções da corrente longitudinal mostram a ocorrência de correntes para NE e para SW em todos os anos avaliados, mas sem uma direção preferencial. Em 2004 e 2005 a direção mais frequente foi NE, e em 2006 para SW.

Durante o verão e primavera houve maior ocorrência de correntes para SW, e durante o outono e inverno para NE. Os meses de fevereiro e março foram os que apresentaram maior bidirecionalidade. A sazonalidade deste comportamento está em acordo com os resultados de Nicolodi et al. (2000), que entre 1996 e 1999 notou predominância de registros totais para SW, interpretadas pelo autor como sendo associadas aos padrões climáticos regionais.

Em todos os meses analisados houve maior concordância do que discordância entre a direção de propagação das ondas e da corrente, indicando uma alta influência das ondas no comportamento da direção da corrente. Em novembro as correntes apresentaram maior número de registros correlacionáveis com o ângulo de incidência das ondulações, assim como nos meses de dezembro, maio e junho. Nos meses de fevereiro, março e abril as frequências foram muito semelhantes, e janeiro foi o único mês que apresentou maior concordância entre a direção do vento e da corrente do que entre as ondas e as correntes. Portanto, os dados apontam as ondas como a principal forçante no padrão de distribuição das correntes longitudinais.

\section{Referências Bibliográficas}

ALMEIDA, L.E.S.B.; ROSAURO, N.M.L. \& TOLDO JR., E.E. Análise Preliminar das Marés na Barra do Rio Tramandaí, RS. In: Anais ABRH SIMPÓSIO BRASILEIRO DE RECURSOS HÍDRICOS, Vitória, ES. v.1, p.560-566, 1997.

ALMEIDA, L.E.S.B.; ROSAURO, N.L.; TOLDO JR., E.E. \& GRUBER, N.L.S. 1999. Avaliação da profundidade de fechamento para o litoral norte do Rio Grande do Sul. In: Anais do SIMPÓSIO BRASILEIRO DE RECURSOS HÍDRICOS, Belo Horizonte, MG. CD-ROM.

ALVAREZ, J.A.; GRÉ, J.C. \& TOLDO JR., E.E. Estudos da praia a nordeste do molhe de Rio Grande - Rio Grande do Sul. Pesquisas, Porto Alegre, 14: 131-147, 1981.

CALLIARI, L.J., HOLAND, T., DIAS M. S., VINZON S., THORTON E. B., STANTON T. P. Experimento Cassino 2005: uma síntese dos levantamentos efetuados na ante-praia e zona de arrebentação. In Congresso da Associação Brasileira de Estudos do Quaternário, 2005. Vitória, ES, ABEQUA, 2005.

FEDDERSEN, F., TROWBRIDGE, J. H. E WILLIAMS III, A. J. Vertical structure of dissipation in nearshore. Journal of Physical Oceanography, 37: 1764 - 1777, 2007.

Fontoura, J.A.S. Hidrodinâmica costeira e quantificação do transporte longitudinal de sedimentos não coesivos na zona de surfe das praias adjacentes aos Molhes da Barra de Rio Grande, RS, Brasil (Aplicação as praias do Cassino, Mar Grosso e adjacências do Molhes Oeste e Leste da embocadura do estuário da Lagoa dos Patos). 2004, 298p. Tese - FURG. Rio Grande, 2004.

Hubertz, J.M. Observations of local wind effects on longshore currents. Coastal Engineering, 10: 275 - 288, 1986.

LANFREDI, N.W. e FRAMIÑAN, M.B. Field Study and Prediction of Longshore Currents, Argentine Coast. Journal of Coastal Reaserch 2(4): 409-417, 1986.

Lisniowski, A. M., 2006. Variação granulométrica nos sedimentos em suspensão transportados longitudinalmente na zona de surfe na praia do Cassino (RS). 2006, 99p. Monografia - FURG. Rio Grande, 2006.

Longuet-Higgins, M.S. Longshore currents generated by obliquely incident sea waves. Journal of Geophysical Research, 75: $6778-6789,1970$.

NICOLODI, J.L; TOLDO JR., E.E. \& GRUBER, N.L.S. Análise da direcionalidade das correntes litorâneas no litoral norte do Rio Grande do Sul. In Anais Semana Nacional de Oceanografia, XIV, 2000, 461- 463.

RUESSINK, B.G, MILES, J.R., FEDDERSEN, F., GUZA, R.T and ELGAR, S. Modeling the alongshore current on barred beaches. Journal of Geophysical Research 106: 451-463, 2001.

TOLDO, E. E., DILLENBURG S. R., ALMEIDA, L. E. S. B., TABAJARA, L. L., MARTINS, R. R. e CUNHA, L. O. B. P. Parâmetros morfodinâmicos da Praia de Imbé, RS. Pesquisas 20 (01) 27-32, 1993.

Tozzi, H.A.M. Influência das tempestades extratropicais sobre o estoque das praias entre Rio Grande e Chuí, RS. 1999, 115p. Dissertação - UFRGS, Porto Alegre 1999. 